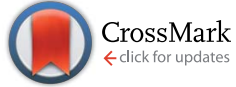

Cite this: Soft Matter, 2015, 11, 2394

Received 23rd September 2014

Accepted 6th February 2015

DOI: $10.1039 / \mathrm{c} 4 \mathrm{sm} 02130 \mathrm{~d}$

www.rsc.org/softmatter

\section{Spider's super-glue: thread anchors are composite adhesives with synergistic hierarchical organization $\uparrow$}

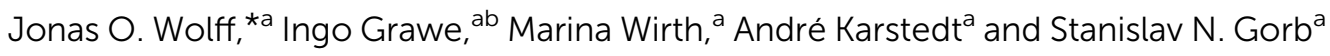

Silk is a key innovation in spiders, fascinating both biologists and material scientists. However, to fulfil their biological function silken threads must be strongly fastened to substrates or other threads. The majority of modern spiders produce a unique and rather unexplored bio-adhesive: the two-compound pyriform secretion, which is spun into elaborate patterns (so called attachment discs) and used to anchor silken threads to substrates. Strong adhesion is achieved on a high variety of surfaces with a minimum of material consumption. Pyriform threads polymerize under ambient conditions, become functional within less than a second and can remain stable for years. They are biodegradable, biocompatible and highly versatile - the adhesion and the overall toughness of the attachment disc can be controlled by spinneret movements on a macroscopic level (ref. 1: V. Sahni et al., Nat. Commun., 2012, 3, 1106, DOI: 10.1038/ ncomms2099). We found that the pyriform thread is a silk fibre that is coated with glue-like cement consisting of aligned nanofibrils, lipid enclosures and a dense, isotropic boundary layer. The threads are spun in a meshwork pattern that promotes stress distribution and crack arresting. Our results demonstrate, that hierarchical organization and fibre embedding may explain the high adhesive strength and flaw tolerance of a structure made by the same, rather simple type of silk glands.
\end{abstract}

\section{Introduction}

Due to its high toughness, spider silk has caught the attention of material scientists and biotechnologists as a possible prototype for developing new biological materials for textile industry and medicine. ${ }^{2-5}$ Silk research concentrates mainly on the major ampullate silk (dragline silk) while other types of silk remain a rather unexplored source of innovation. By gene duplication the more advanced araneomorph spiders evolved up to eight different types of silks, ${ }^{6-8}$ varying from ultra-tough nanofibres $\left(\right.$ aciniform silk) ${ }^{9}$ to viscoelastic glycoprotein based glue droplets (aggregate secretion). ${ }^{10}$

Pyriform silk is used to agglutinate silken threads or to affix them to substrates. It can produce strong adhesion, even on surfaces with a low free surface energy, such as Teflon. ${ }^{11}$ The pyriform glands are comparably small with a pear-shaped (name) lumen and a short duct opening into the nozzle-like spigot. $^{12,13}$ These appear in large clusters on the anterior (first)

${ }^{a}$ Department of Functional Morphology and Biomechanics, University of Kiel, Am Botanischen Garten 1-9, D-24098 Kiel, Germany. E-mail: jwolff@zoologie.uni-kiel.de ${ }^{b}$ Department of Mechanical Engineering, Westphalian Institute for Biomimetics, University of Applied Sciences, Münsterstrasse 265, 46397 Bocholt, Germany

$\dagger$ Electronic supplementary information (ESI) available: Comparison of the results of adhesion experiments on differently treated glass with those on Teflon. Characterization of test surfaces (water contact angle and mean roughness). See DOI: 10.1039/c4sm02130d of, typically, three spinneret pairs next to the major ampullate gland opening. This arrangement facilitates the fast attachment of the dragline. ${ }^{14}$ The pyriform gland exhibits two histochemically distinct parts each with a secretory cell type..$^{12,15}$ The distal half of the gland produces the PySp spidroins (silk proteins), the proximal half secretes a colloidal fluid containing, as yet unidentified, acidic proteins and hydrocarbons..$^{15}$ The secretory products form two phases in both the gland lumen and the duct. ${ }^{13,15}$ Morphologically and histochemically these glands are very similar to the single silk gland type found in ancient lineages of spiders whose threads adhere by means of an acidic protein coating. ${ }^{16}$ Pyriform silk may thus have a very early origin and been highly optimized throughout evolution. With the evolution of insect flight, spiders began to spread out from their ground habitats into vegetation and to build aerial webs. ${ }^{8,17}$ As plants often exhibit complex surfaces with anti-adhesive properties for staying clean and deterring herbivores, ${ }^{18,19}$ among spiders there might have been a strong selective pressure for the best glue, which made the best foraging sites accessible. ${ }^{11}$

The macroscopic structure of the attachment discs is the result of a highly conserved spinneret movement program creating numerous parallel loops of crossing silk fibres. ${ }^{14,20}$ In the central part the dragline is glued, elevated and thus not in contact with the substrate attachment. This provides certain flexibility and a more homogenous stress distribution within the structure. ${ }^{11}$ Spiders may be able to spin attachment discs with different adhesion and overall toughness: ${ }^{1}$ through the 
coordinated action of the anterior spinnerets discs can be created that are attached to the substrate only at their margins and thus be easily detached by a small impact of the mobile prey. This kind of disc is used in unique traps called gumfoots. ${ }^{1}$ Since attachment discs are frequently used by spiders they must have been selected for high economy in the course of evolution. The attachment disc of an adult (body mass $0.6-0.8 \mathrm{~g}$ ) golden orb weaver (Nephila senegalensis) can hold 4-6 times its body weight when applied to a smooth glass surface, ${ }^{11}$ while containing only $2-10 \mu \mathrm{g}$ ( $\sim 0.001$ per cent of spider weight) of material. Hence, the usage of pyriform silk by spiders may show means of applying glue in a way that minimises the use of material. ${ }^{21}$ Furthermore, the intrinsic composite structure of the attachment disc can be a great source of inspiration for the engineering of nanocomposite and light weight materials. ${ }^{11}$ It is also noteworthy that pyriform silk may have a high potential as a natural glue or as possible component of synthetic biomaterials for medical applications and future green technologies. ${ }^{22}$ The first progress made along this line of research is the recent identification, isolation and cloning of pyriform spidroins. ${ }^{22,23}$ However, a basic understanding of the mechanism of how the attachment disc functions is still lacking. Here we present the first comprehensive study on pyriform glue which (1) integrates ultra structural and micromechanical analysis, (2) reveals some synergistic effects in its hierarchical organization, and (3) gives insight into the structure-function relationships of the discs.

\section{Results}

Spider attachment discs are divided into four functional parts (Fig. 1c): (1) the substrate cementation which can be regarded as a thin film (baseplate) (Fig. 1e); (2) a network of pyriform fibres between the baseplate and the cemented dragline (bridge) (Fig. 1d); (3) an envelope of pyriform fibres cementing the dragline (conjunction) and (4) the major ampullate silk threads anchored by the attachment disc (dragline).

The pyriform thread is a twofold compound material, a fibrous cement with an embedded fibre (Fig. $1 \mathrm{~g}$ and $\mathrm{h}$ ). By means of electron microscopy we found that the main fraction of the cement material is made up of nanofibrils (Fig. $1 \mathrm{~h} ; 2$ ), consisting of $20-30 \mathrm{~nm}$ sized electron dense globular proteins connected by thin strains of a material with less electron density (Fig. 2e). This is very similar to the structure of the secretion before extrusion. ${ }^{15}$ The cement nanofibrils are regularly arranged parallel to one another and to the spidroin fibre. They form stacked monolayers that get partially separated by strong shear forces during freeze fracturing (Fig. 2b). In transverse fractures the cement forms a rather smooth breaking edge with a regular arrangement of the globular proteins (Fig. 2a). In contrast, the embedded spidroin fibres exhibit a corrugated breaking edge indicating a more amorphous structure and higher toughness of the material (Fig. 2a). At the interface towards the substrate and the environmental medium (air) a thin $(\sim 10 \mathrm{~nm})$ electron dense layer forms (Fig. 2a, c-e) which might consist of small, highly polar proteins that act as an intermediate agent between the substrate and the silk cement. In contrast to the cement and spidroin fibre, this part seems to be isotropic as it breaks in various directions. Surprisingly, when deep frozen at $-140{ }^{\circ} \mathrm{C}$ in liquid nitrogen for the purpose of freeze fracturing the attachment discs remained flexible and were hardly breakable, indicating a low content of unbound water.

We studied the spinning process using reflection interference contrast microscopic high speed videography (RICM-HSV) and Cryo scanning electron microscopy (Cryo-SEM). We found that the material is a low viscosity fluid, which is less organized when extruded (Fig. 3b). The embedded silk fibre is already differentiated against the glue phase and appears to be rather solid, but elastically deformable (Fig. 3f-l). The thread is extruded with a velocity of $5-9 \mathrm{~mm} \mathrm{~s}^{-1}$. Within less than a second the cement is dried and no longer changes its shape when occasionally touched by a spider's cuticular structure. At high spinning activity, a condensation of minute droplets was observed on the glass substrate between the fibres (Fig. $3 \mathrm{~m}$ and $\mathrm{n}$ ), which we interpreted as water evaporated from the rapidly drying cement. If the threads are not directly applied onto the substrate, but with a short delay after extrusion, beads-on-astring structures (BOAS) occasionally occurred (Fig. 3d). This is an indication, that the drying glue behaves like a viscoelastic fluid. ${ }^{24}$ The spidroin thread is clearly embedded and does not directly adhere to the substrate, which is indicated by its occasional dislocation within the glue coating shortly after its contact to the substrate (Fig. 3f-l). Thus, the assumption of previous authors about strong interaction between the PySp thread and the substrate 22,23 is not supported by our data.

Once applied, attachment discs remain stable for a long period of time: we found that attachment disc samples of a $N$. senegalensis individual (on glass) stored for a year still retained, on average, three-fourths of the attachment force of freshly harvested ones (fresh: $39.8 \pm 8.9 \mathrm{mN}, n=23$; aged: $28.5 \pm$ $11.2 \mathrm{mN}, n=13)$.

The adhesion of the pyriform glue outweighs the inner strength of the attachment disc on a smooth glass surface and the structure breaks internally ( $87 \%$ of 32 tests) or at the dragline $(13 \%)$. When the glass is treated with APTES (3-aminopropyl-triethoxysilane), which increases its hydrophobicity, the pyriform glue adheres less strongly. This is indicated by partial delaminating of the attachment disc during the tensile test (69\% against $0 \%$ on untreated glass of each 32 tests) and reduced detachment forces (Fig. 4a). On glass treated with DMDCS (dichlorodimethylsilane), which forms a strongly hydrophobic film on the surface, spiders are unable to attach their pyriform threads. This indicates the important role of hydrogen bonds in the adhesion of pyriform cement. Monolayers of water usually present on uncoated glass (and on most other surfaces ${ }^{25,26}$ ) may act as a coupling agent and support adhesion.

On PTFE-foil (Teflon), ${ }^{11}$ which exhibits a similar water contact angle than that of the DMDCS treated glass, adhesion is significantly lower than on the APTES treated glass, but still sufficient to hold the body weight of the spider. The better attachment to Teflon than to DMDCS treated glass might be explained by much stronger nano roughness of Teflon foil than of DMDCS treated glass (see ESI S1†). 


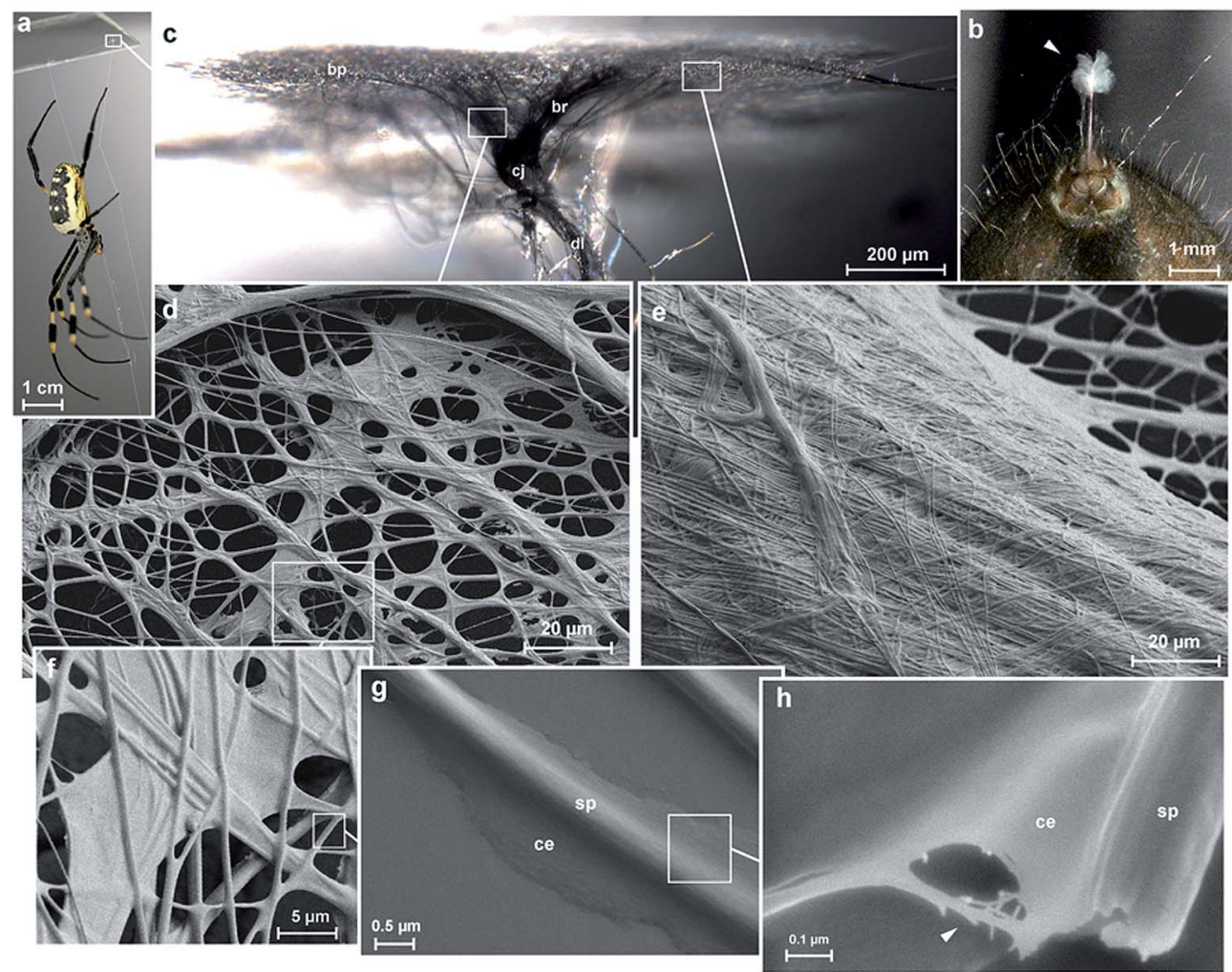

Fig. 1 Hierarchical structure of spider silk anchors. Spiders, such as the golden orb weaver (Nephila senegalensis), can secure themselves on smooth glass by means of silk (a). The tough safety thread (dragline), made of major ampullate silk, is attached to the slide with pyriform silk (b). It is spun in an elaborate 3D-pattern called the attachment disc (c). It consists of an apical part where the dragline (dl) is glued (conjunction, cj), an intermediate network of pyriform threads (bridge, br) (d) and the basal substrate cementation (baseplate, bp) (e). The pyriform threads are applied in layers with shifted angles (f) and consist of two phases: a spidroin (silk protein) fibre (sp) and a glue coating (cement, ce) which is fluid-like just after extrusion and dries rapidly after application (g). The cement consists of aligned nano-fibrils, as seen at rupture sites (h).

\section{Discussion}

The adhesion of silk threads is substantial for their function in protection, prey capture, locomotion or reproduction, as these always implicate an interaction with substrates or other previously spun threads. In insects and spiders silks have been shown to be coated with an acidic protein (sericin in Bombyx mori $^{27}$ ) or a glycoprotein (spider dragline ${ }^{28}$ ). The thin draglines of small spiders can stick to smooth surfaces such as glass. However, the attachment is not sufficient to hold the body weight of the spider. ${ }^{29}$ Fastened with a pyriform anchor it can securely attach a spider even on Teflon (PTFE), which is wellknown and applied for its extremely reduced surface interaction with other substances. ${ }^{\mathbf{1 1}}$ Our results show an elaborate hierarchical structure of attachment discs and the combination of materials with different properties that may complement one another. In the following we discuss the role of different hierarchical levels from the molecular one to the macroscopical one and how these may interact synergistically.

\section{Molecular structure and ultra structure}

Previous chemical analysis of the pyriform proteinous fraction showed an extraordinarily high content of polar and charged amino acids, ${ }^{15,23,30,31}$ which may result in strong interactions within the dried secretion film and with the substrate. The highly polar regions within pyriform spidroins also support selfassembly of the silk fibre ${ }^{23}$ and water-solubility, ${ }^{31}$ both being desirable properties for use in artificial spinning systems. Further, carbohydrate components were found in the cement fraction, ${ }^{15}$ perhaps indicating the presence of glycoproteins. These play a universal role in adhesive cementation ranging from cells ${ }^{32}$ to marine organisms. ${ }^{33}$ Our ultra structural analysis showed that there are inclusions of, presumably, lipids within the cement. These could play a role as surfactants, improving the wettability of hydrophobic surfaces. We found that the cement consists of aligned heteromerous nano-fibrils of repetitive globular and fibrillar parts. Nano-fibrils with such geometry are hypothesized to provide a high resistance against bending, tensile and shear stresses, thanks to interlocking. ${ }^{34,35}$ This is of high relevance for the stability of the attachment under load.

The ultra structural analysis of freeze fractured pyriform fibres showed that both phases, the fibre and the cement, differ fundamentally in their fracture mechanics, indicating a difference in their mechanical properties. Previous amino acid sequence analysis of PySp showed that these spidroins are less organized but rather amorphic, ${ }^{15,23,31}$ which is confirmed by the results of our ultrastructural analysis. Such structure usually 

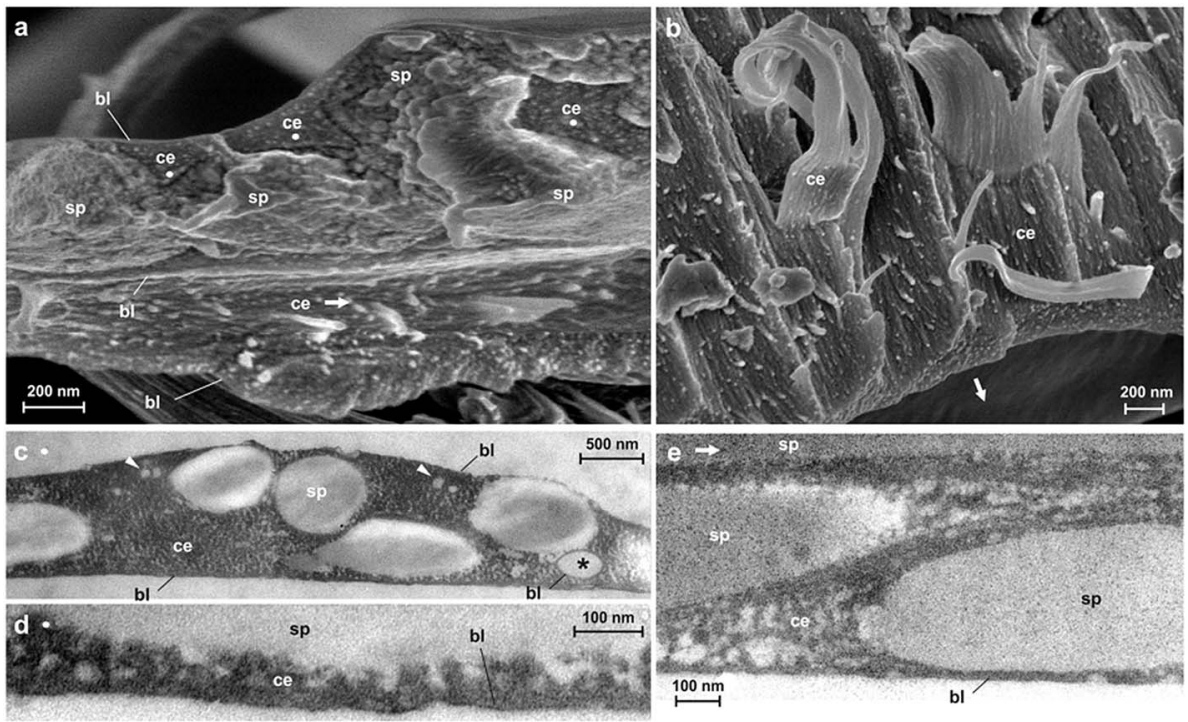

Fig. 2 Ultrastructure of pyriform silk. (a-b) Cryo SEM micrographs of freeze fractured pyriform threads of Nephila senegalensis. (a) A transverse fracture of a thread crossing visualizing the different phases and its supramolecular organization. The pyriform cement (ce) consists of regularly arranged nano-fibrils aligned in the same direction as the embedded spidroin thread (sp) which consists of a less ordered material that, in contrast, does not show a smooth breaking edge. At the interface between the pyriform thread and the substrate the medium or earlier spun (already dried) threads show a thin boundary layer (bl), presumably a monolayer of regularly arranged macromolecules. Arrows and dots indicate orientation of polymer fibrils (dots indicate orientation perpendicular to the image plane). (b) A horizontal fracture, where spidroin fibres are ruptured, showing that the cement nanofibrils form layers. (c-e) TEM images show that cement fibrils are heteromeric polymer chains consisting of alternating electron dense and electron translucent parts. Spidroin fibres appear amorphous and less electron dense. The boundary layer has a high electron density. Occasionally droplets (presumably lipids, arrowheads) and air inclusions (asterisk) are found in the cement fraction.

indicates high ductility. Material heterogeneity (although less pronounced) is also known from the major ampullate silk of spider draglines. These fibres consist of two different spidroins (MaSp1 and MaSp2). The MaSp1, located in the outer core of the fibre, exhibits a semi-crystalline structure, providing high strength. ${ }^{36}$ In the inner core, there is an additional protein, the MaSp2, which, in contrast, exhibits a disordered structure due to a high content of glycine, thereby providing high extensibility. ${ }^{36}$ Thus, due to the synergistic effect of both polymers the fibre exhibits high toughness. An analogy from artificial materials is the recent development of Engineered Cementitious Composites (ECC), which demonstrates that a strong, yet brittle material such as concrete can get tough and flaw tolerant through the inclusion of polymer fibres, because crack propagation is stopped at the interface between both materials. ${ }^{37}$ The two fold compound structure of pyriform silk may reinforce the material in a similar way.

\section{Micro- and mesostructure}

In a pyriform fibre the glue fraction usually constitutes less than half of the secretion. This might be substantial to quickly arrest the thread when applied to the surface. The glue coating must be thick enough to fill small cavities on a rough surface in order to generate a high contact area, crucial for adhesion..$^{38}$ On the other hand, it must be thin enough to prevent cohesion failure when drag forces are acting on the embedded fibre as long as the glue is in a fluid state (as indicated by partial displacement at turning points found during RICM-HSV recordings; Fig. 3f-i).
It should further form a thin film on the substrate from which the solvent can quickly evaporate. A thin film exhibits high adhesive properties due to high elasticity (even if the material is relatively stiff).$^{39}$ Depending on where the force is applied, the peeling line of a thin film (crack propagation zone) can be linear (uni-directional) or axisymmetric (multi-directional). The highest pull-off forces are achieved in the case of multidirectional peeling because the peeling line continuously increases, causing an increase in detachment resistance. ${ }^{40}$

On smooth substrates with reduced adhesion (silanized glass) we often observed radial symmetrically-oriented crack propagation (Fig. $4 \mathrm{~d}-\mathrm{m}$ ). Linear peel-offs only occurred in single threads at the margin of the attachment discs, where the pyriform threads are less dense and less interconnected. The propagation of cracks was significantly hindered by the material heterogeneity of the composite, leading to discontinuous peel-offs and high increases of detachment forces (Fig. 4p). Crack propagation is often stopped at threads arranged perpendicular to the crack propagation direction (Fig. 4n and o). Cracks are thus guided by the strong anisotropy (directionality) of the pyriform silk on both ultrastructural (cement nanofibrils) and microstructural (embedded spidroin fibres) levels. Due to the crossover arrangement of pyriform fibres in the attachment disc basal plate (determined by spinneret movements during attachment disc spinning), cracks are trapped between thread interconnections and their propagation is stopped. Because the creation of a new crack demands significantly more energy than its propagation, the force necessary to detach the attachment disc rises. This principle is furthermore important for flaw tolerance. Most natural surfaces that are the 

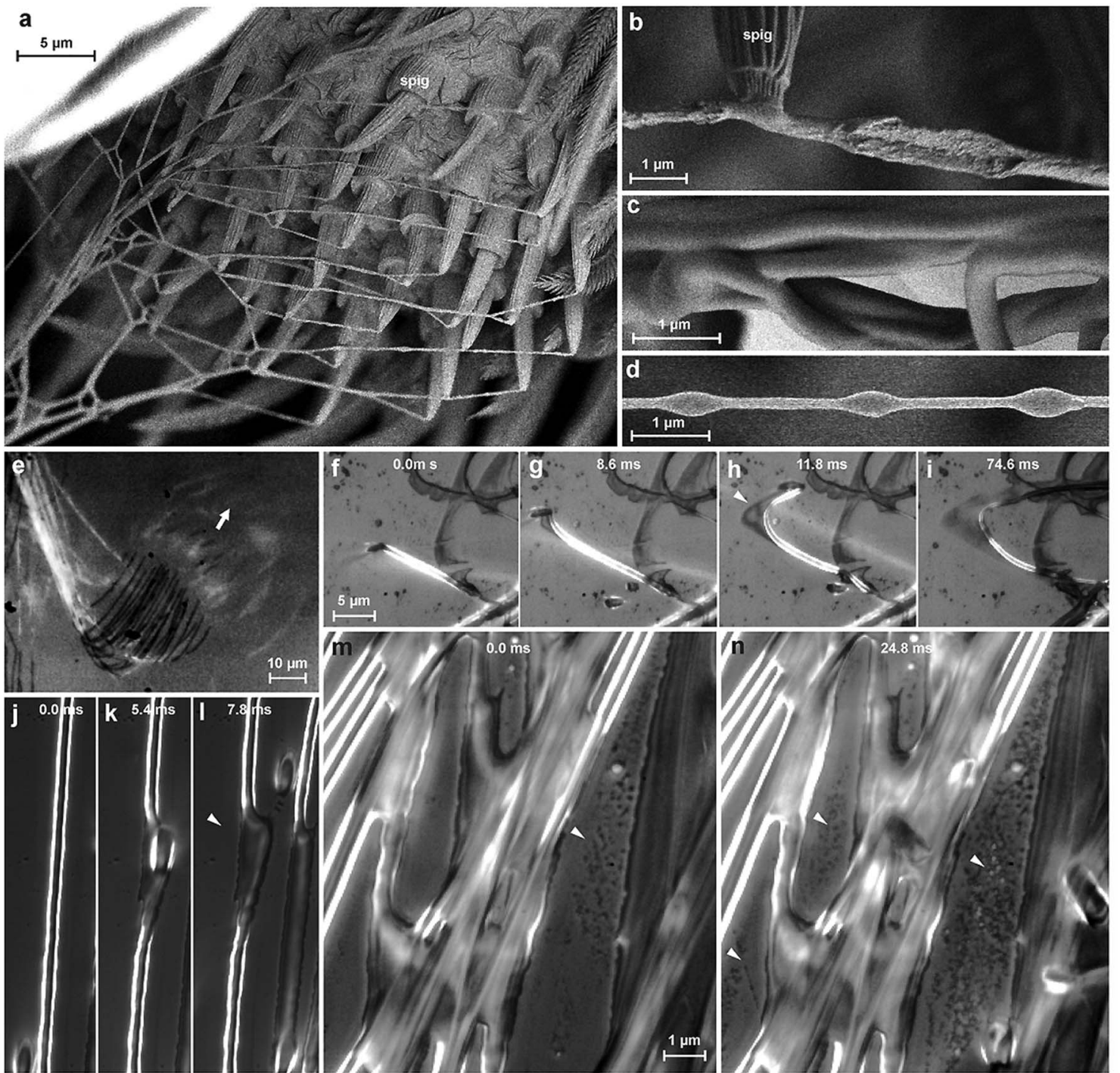

Fig. 3 Spinning of pyriform silk. (a) During attachment disc spinning, pyriform silk is extruded by numerous spigots (spig) of the anterior lateral spinnerets, here in the cribellate orb weaver (Uloborus plumipes). The silk is liquid, when extruded, as indicated by the irregular surface of the thread (b). The same threads have a smooth surface at a greater distance from the spinneret, indicating post-spinning flow (c). Occasionally beads-on-a-string structures (BOAS) occurred, an indication that the drying glue behaves like a viscoelastic fluid (d). The application of the attachment disc onto a glass slide can be studied by means of RICM-HSV (view from below the glass slide), where direct contact appears darkened (a single spinneret of $U$. plumipes in action in (e), with an arrow indicating spinneret movement). Sequence (f-i) shows the application process in Nephila senegalensis at a turning point, showing that the internal spidroin thread is pulled over some distance at the distal point of the loop, smearing the cement phase over the substrate. This indicates that the thread is already solidified, while the cement is still in a fluid state. This is further supported by the observation that crossing (in this case: non-secreting) spigots take the thread with them over a short distance ( $\mathrm{j}-\mathrm{l}$ ). When pyriform threads are applied at a high density, condensation of a liquid takes place on the glass in between ( $\mathrm{m}$ and $\mathrm{n})$, an observation which assumes water evaporation from the drying silk.

target of attachment disc application are highly unpredictable and exhibit excessive heterogeneity in surface topography and/or surface chemistry. This means that flaws in the adhesive cannot be prevented. Cracks can easily be induced and propagate from such defects. By proper crack arresting the effect of adhesion defects is suppressed.

\section{Macrostructure}

The macrostructure of the attachment disc may substantially support energy dissipation, thus reducing stress concentration, because of the interconnections of pyriform threads in the bridge (and the baseplate). Because both the dragline and the bridge are not rigid, but rather ductile, energy might be dissipated by elastic and plastic deformation. The bi-axial symmetry of the attachment disc (determined by the arrangement of the paired spinnerets) leads to simultaneous peeling on opposite sites of the attachment disc, as observed in RICM-HSV peel off tests from APTES treated glass (Fig. 4b and c). Thus, the peeling angle is kept low, promoting stress distribution at the peeling edge and therefore higher pull-off forces. ${ }^{41}$ Energy dissipation 

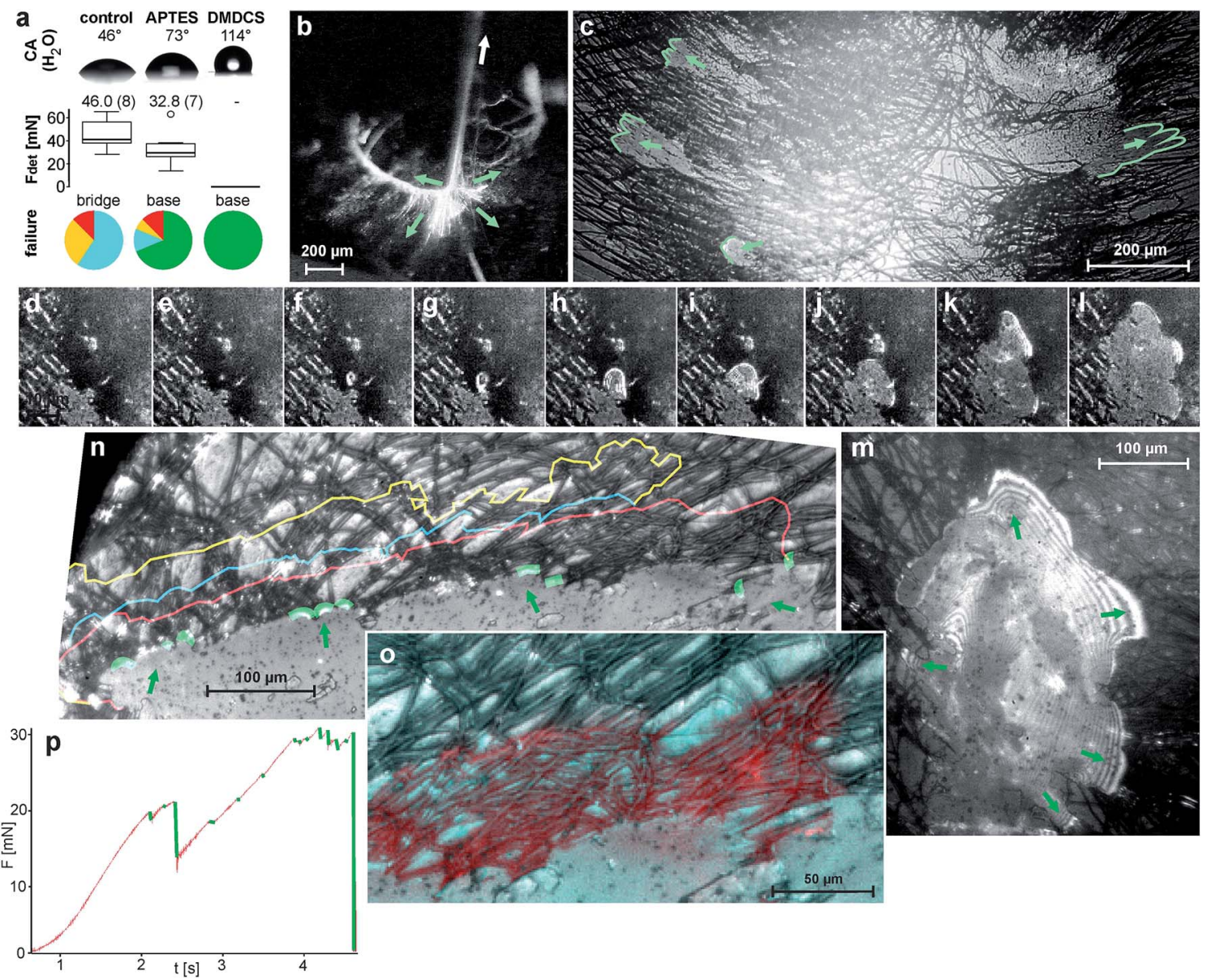

Fig. 4 Fracture mechanics of pyriform silk discs. (a) Results of tensile tests of $N$. senegalensis attachment discs spun on untreated and silantreated glass resulted in different detachment forces and failure modes. Above, the contact angle (CA) measurement of water droplets on the substrates demonstrates their differences in hydrophilicity. Box plots give the median and variance of detachment force (Fdet) data. Numbers above box plots give the mean values (in brackets number of individuals tested). Pie charts in the bottom indicate the proportion of failure modes, whereby red symbolizes dragline failure, yellow conjunction failure, blue bridge failure and green baseplate failure (total delamination) (for details see ref. 11). These results indicate the importance of hydrogen bonds in pyriform silk adhesion. (b) When the dragline is pulled (arrow), various pyriform fibres of the bridge are put under tension (green arrows depict direction of acting tensile stress). (c) Due to their radial arrangement, stress is distributed to opposite sides of the symmetrical baseplate, leading to a simultaneous peel-off when spun on APTES-treated glass (green lines mark the peeling edges). (RICM-HSV frames, view from below the glass slide) $(d-m)$ A stressed bridge fibre may induce a crack at the interface (arrowhead), which is initially circular and often propagates radial symmetrically first. The stress is distributed at the peeling edge, as indicated by interference stripes in the RICM image that occur when the separation between substrate and silken film is very low (m). (n) Crack arresting indicated by coloured lines. (O-p) Crack propagation is often stopped at crossing fibres. (o) A detail, with the next step failure marked in red. Please note that the crack propagation is stopped along transverse fibres. ( $p$ ) The force-time curve during a tension test on APTES-treated glass shows that cracks (green marked force drops) are quickly arrested, followed by a further rise in the force. Shortly before the final rupture, the frequency of crack initiation increases because stress approaches the maximum that the attachment disc can withstand.

and stress distribution in both the macrostructure of the attachment disc and at the peeling edges (crack propagation zone) results in low stress actually acting on the substratecement interface. This might explain why the attachment discs achieve considerable attachment strength even if the substrate bonding is relatively weak, as on Teflon.

\section{Conclusion}

Spider attachment discs demonstrate the synergistic effect of structural parameters on different hierarchical levels from the molecular to the macroscopic, which altogether provide a high adhesive strength and toughness on unpredictable surfaces at an absolute minimum use of material (Fig. 5). On the molecular level the high content of polar and charged side chains leads to strong interaction with substrates (adhesion) and within the material (cohesion). On the ultrastructural level aligned heteromeric nanofibrils lead to high anisotropy and strength. The composite structure of two phases (spidroin fibre and cement coating) creates toughness and anisotropy on the microstructural level and introduces a material heterogeneity, stopping crack propagation. The meshwork pattern, by overlays of pyriform threads applied in different angles, promotes stress distribution and flaw tolerance by crack arresting (mesostructural level). Finally, on the macrostructural level, the separated 

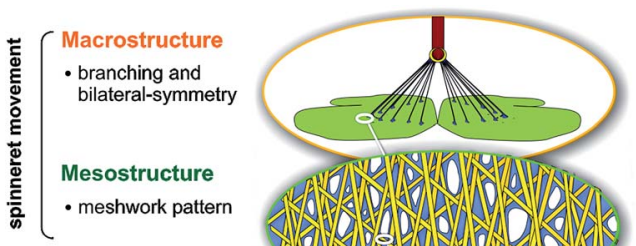

- resistance against bending / torsional stresses

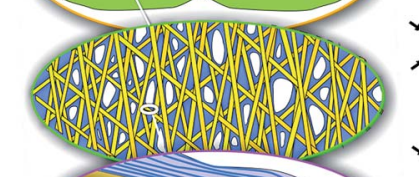

stress distribution
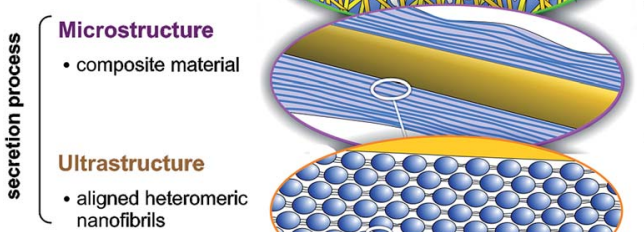

crack arresting

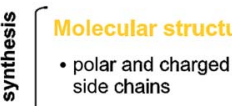

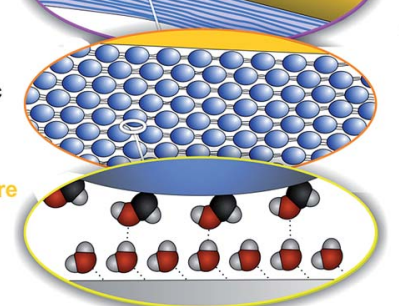

$\rightarrow \quad$ toughness

anisotropy

strength

$\rightarrow$ adhesion

$\rightarrow$ cohesion

Fig. 5 Hierarchical organization and functional correlates. Schematic illustration and description of the spider attachment disc structure summarizing the main hypotheses of this study. For further explanation, see text.

cementation of substrate and dragline, as well as bi-lateral symmetry, supports load energy dissipation and a reduction of sensitiveness towards bending and torsional stresses. These structure-function relationships are substantial for the basic understanding of attachment disc function and may significantly support the exploitation of this unique bioadhesive in biotechnological and biomimetic approaches, for both medical and technical applications. It further illustrates that an elaborate secreted structural material, such as silk, must be studied not only on the biochemical but also on the physiological and especially organismic (behavioural, phylogenetic and ecological) level. The ecological demands and phylogenetic burden that determine and constrain the evolution of a biological material, must also be taken into account to understand and extract principles of a structure-function relationship.

\section{Material and methods}

\section{Animals and harvesting of attachment discs}

Ten living individuals of the golden orb weaver Nephila senegalensis Walckenaer 1842 (Nephilidae) were obtained from a laboratory stock of the Department of Ethology, University of Hamburg, Germany. Spiders were kept in reversed $500 \mathrm{ml}$ plastic cups (with roughened walls and an apical hole closed with a piece of plastic mull) at $28-30{ }^{\circ} \mathrm{C}$ and $60-70 \%$ relative humidity. They were sprinkled daily with water and fed weekly with juvenile locusts (Locusta migratoria) obtained from the local pet shop. For snap freezing experiments of spinning spiders, small cribellate orb weavers (Uloborus plumipes Lucas 1846 (Uloboridae)) were collected in the greenhouses of the local botanical garden (this species was used for this particular experiment because of the restricted space in the set-up and its very frequent production of attachment discs). In high speed

video recordings (HSV) of attachment disc production (see methodology below), we found no differences between representatives of Nephila and Uloborus, despite their different body sizes.

Attachment discs were harvested by holding the substrate sample with a long pair of forceps. The spider was induced to crawl onto the substrate by slightly touching the animal on the hind legs. When spiders were left sitting upside down on the substrate (usually the edges of the slides were grasped with the claws) they often secured themselves by means of an attachment disc. Spinning could also be induced by slightly shaking or blowing at the spider. The spider was given the freedom to move forward, without forcing it, until the dragline had a proper length. Then the dragline was cut, with a fine pair of scissors, at about $3 \mathrm{~cm}$ above the attachment disc. Substrate separated samples for ultrastructural analyses were obtained by letting the spider spin onto a piece of Teflon from which the attachment disc could be completely delaminated.

Samples of attachment discs delaminated from Teflon were weighed with using an ultra balance (UMX2, Mettler-Toledo Inc., Columbus, OH, USA) (dragline was cut off above the attachment disc prior to weighing).

\section{Light microscopy (LM) and high speed videography (HSV)}

Macro-morphology of attachment discs were studied using standard transmission light microscopy (phase contrast mode). Spinning of attachment discs was studied using reflection interference contrast microscopic high speed videography (RICM-HSV). This was conducted by means of an inverted microscope (AXIO Observer.A1, Carl Zeiss AG, Oberkochen, Germany), operated with coaxial light and a beam splitter reflecting the image onto the sensor of a high speed video camera (Fastcam SA 1.1, Photron Inc., San Diego, CA, USA). A $40 \times / 0.65$ lens and a $100 \times / 1.4$ oil immersion lens were used. Thin, cleaned (for cleaning protocol see below) cover slips were mounted on a Plexiglas slide having a median hole, fixed with double sided tape to reduce the bending of the slide under load possibly leading to unfocused recordings. Spiders were set onto the slide with the posterior end of the abdomen placed into the hole with the underlying cover slip and recordings were made with 5000 frames per second using continuous recording and post-triggering.

\section{Electron microscopy (EM)}

Attachment disc samples on pieces of cover slips and delaminated samples were fixed on stubs with conductive doublesided carbon tape and sputter coated with $12 \mathrm{~nm}$ AuPd. Samples were imaged in a Hitachi S 4800 scanning electron microscope (SEM) (Hitachi Ltd., Tokio, Japan) at an acceleration voltage of $3.0 \mathrm{kV}$.

Uloborus spiders were fixed on the tip of a bound piece of metal wire by means of a droplet of two compound dental wax (polyvinylsiloxane, Coltène/Whaledent AG, Altstätten, Switzerland). The wire piece was glued onto a SEM specimen holder beside a small wooden block with an attached piece of tree leaf on an $80^{\circ}$ sloped edge, serving as a spinning substrate. By 
bending the wire, the spider was brought into a position with the spinnerets pointing upwards and being close enough to the wood block to reach the leaf. When spiders started to spin an attachment disc, the entire specimen holder was immediately put into liquid nitrogen and the deep frozen sample was transferred into an S 4800 SEM equipped with a Gatan ALTO2500 Cryo system (Gatan Inc., Abingdon, UK), sputtered with 10 $\mathrm{nm} \mathrm{Au}-\mathrm{Pd}$ and viewed in the SEM with the stage cooled up to $-120{ }^{\circ} \mathrm{C}$.

For freeze fracturing, Nephila attachment discs, initially peeled off the Teflon substrate, were vertically glued onto a specimen holder with Tissue-Tek ${ }^{\circledR}$ compound, shock frozen in liquid nitrogen and transferred to the SEM Cryo system. Fracturing was executed by cutting and scraping the sample with a scalpel blade, mounted on a moveable metal stick within the super-cooled SEM prechamber. Then the samples were directly sputter coated with $8 \mathrm{~nm}$ AuPd and viewed at 3.0 and $10.0 \mathrm{kV}$.

For transmission electron microscopy (TEM) attachment disc samples were collected on ACLAR®-foil (Plano $\mathrm{GmbH}$, Wetzlar, Germany), which is inert against the chemicals used in the TEM sample preparation process. Samples were fixed with $2.5 \%$ gluteraldehyde in PBS buffer and 1\% osmium tetroxide, dehydrated in a series of ethanol solutions of increasing concentration and embedded in Epon resin. After Epon polymerization, the ACLAR®-foil was peeled off and a second layer of Epon was applied on the side where the foil was detached. $40 \mathrm{~nm}$ ultrathin sections were made with a Leica EM UC7 ultramicrotome (Leica Microsystems GmbH, Wetzlar, Germany), mounted on copper grids and post stained with $1 \%$ uranyl acetate $(20 \mathrm{~min}$ ) and $2 \%$ lead citrate $(7 \mathrm{~min})$, rinsed in $\mathrm{CO}_{2}$ free aqua bi-dest, an observed in Tecnai G2 Spirit (FEI Corp., Hillsboro, USA).

\section{Tensile tests}

Glass slides (Carl Roth GmbH \& Co. KG, Karlsruhe, Germany) were cleaned by rinsing with acetone, ethanol and twice with distilled water and rubbed with KimWipe lab tissues. Some slides were bathed in 1\% APTES ((3-aminopropyl)triethoxysilane) solution in a mixture of acetone and distilled water, rinsed three times with acetone and dried in an oven at $110{ }^{\circ} \mathrm{C}$ for $1 \mathrm{~h}$. Other slides were exposed to vapours of DMDCS (dichlorodimethylsilane) by placing them in sealed Petri dishes with droplets of concentrated DMDCS solution for one night and then rinsed with acetone several times until excessive, unbound DMDCS was removed. The contact angle of silantreated and untreated glass slides was measured with DataPhysics OCA 20 (DataPhysics Instruments GmbH, Filderstadt, Germany) using $500 \mu$ l droplets of aqua bi-dest.

Freshly harvested Nephila attachment discs were tested by pulling on the upstream (last spun, previously directed towards the spider) dragline. Substrate slides were placed onto a lab boy and the dragline was fixed at a length of $10 \mathrm{~mm}$ onto the cantilever of a load cell force transducer with $20 \mathrm{~g}$ force range (World Precision Instruments Inc., Sarasota, FL, USA) by means of a molten beeswax droplet. The force transducer was mounted on a micromanipulator (DC3001R with controller MS314, World
Precision Instruments Inc., Sarasota, FL, USA), which provided constant $\left(200 \mu \mathrm{m} \mathrm{s}^{-1}\right)$ vertical movement. Force curves were recorded with AcqKnowledge 3.7.0 software (Biopac Systems Ltd, Goleta, CA, USA). Tension tests were simultaneously filmed using a Firefly pro GT 800 camera (Firefly Global, Belmont, USA) for the analysis of failure modes. The following failure modes were distinguished: (1) dragline failure (dragline brakes above or at the attachment disc), (2) conjunction failure (failure at the interface of the dragline-pyriform envelope), (3) bridge failure (breakage of the attachment disc above the substrate) and (4) baseplate failure (partial or total delamination of the attachment disc). Pull-off forces were taken as the highest force peaks measured during attachment disc pulling. In total, attachment discs of 8 individual spiders were tested with at least 3 attachment discs of each individual on each substrate. Data was analyzed using $\mathrm{R}$ software (R Core Development team, http:// www.r-project.org/) whereby means of all attachment discs of the same individuals were taken as individual data points in comparative analysis.

Single tensile tests of attachment discs spun on APTEStreated glass slides were filmed with RICM-HSV (method described above) using $10 \times, 20 \times$ and $40 \times$ lenses and frame rates of 250, 2000 and $5000 \mathrm{fps}$.

\section{Author contributions}

J.O.W., I.G., and S.N.G. conceived and designed the experiments. J.O.W., M.W., I.G. and A.K. performed the experiments and analyzed the data. J.O.W. and S.N.G. wrote the paper.

\section{Funding}

This work was supported by the German Science Foundation (DFG) to S.G. (GO 995/10-1) and the German National Merit Foundation (Studienstiftung des Deutschen Volkes) to J.O.W.

\section{Competing interests}

The authors declare no competing financial interests.

\section{Acknowledgements}

We thank Prof. Jutta Schneider (University of Hamburg) for providing experimental animals. Theresa Gödel is acknowledged for characterizing the surface of substrates used in experiments by the means of atomic force microscopy. Thanks to Lars Heepe (University of Kiel) for constructive discussion and paper suggestions on adhesion physics. Victoria Kastner (Max Planck Institute for Developmental Biology, Tübingen) provided linguistic corrections of the manuscript. Two anonymous reviewers improved the manuscript by worthy comments and suggestions.

\section{References}

1 V. Sahni, J. Harris, T. A. Blackledge and A. Dhinojwala, Cobweb-weaving spiders produce different attachment 
discs for locomotion and prey capture, Nat. Commun., 2012, 3, 1106, DOI: 10.1038/ncomms2099.

2 F. Vollrath and D. P. Knight, Liquid crystalline spinning of spider silk, Nature, 2001, 410, 541-548, DOI: 10.1038/ 35069000.

3 M. B. Hinman, J. A. Jones and R. V. Lewis, Synthetic spider silk: a modular fiber, Trends Biotechnol., 2000, 18, 374-379, DOI: 10.1016/S0167-7799(00)01481-5.

$4 \mathrm{~K}$. Schacht and T. Scheibel, Processing of recombinant spider silk proteins into tailor-made materials for biomaterials applications, Curr. Opin. Biotechnol., 2014, 29, 62-69, DOI: 10.1016/j.copbio.2014.02.015.

5 A. Rising, Controlled assembly: A prerequisite for the use of recombinant spider silk in regenerative medicine?, Acta Biomater., 2014, 10, 1627-1631, DOI: 10.1016/ j.actbio.2013.09.030.

6 F. Vollrath, Spider Webs and Silks, Sci. Am., 1992, 266, 70-76.

7 T. A. Blackledge and C. Y. Hayashi, Silken toolkits: biomechanics of silk fibers spun by the orb web spider Argiope argentata (Fabricius 1775), J. Exp. Biol., 2006, 209, 2452-2461, DOI: 10.1242/jeb.02275.

8 A. M. T. Harmer, T. A. Blackledge, J. S. Madin and M. E. Herberstein, High-performance spider webs: integrating biomechanics, ecology and behaviour, J. R. Soc., Interface, 2011, 8, 457-471, DOI: 10.1098/rsif.2010.0454.

9 C. Y. Hayashi, T. A. Blackledge and R. V. Lewis, Molecular and mechanical characterization of aciniform silk: Uniformity of iterated sequence modules in a novel member of the spider silk fibroin gene family, Mol. Biol. Evol., 2004, 21, 1950-1959, DOI: 10.1093/molbev/msh204.

10 V. Sahni, T. A. Blackledge and A. Dhinojwala, Viscoelastic solids explain spider web stickiness, Nat. Commun., 2010, 1, 19, DOI: 10.1038/ncomms1019.

11 I. Grawe, J. O. Wolff and S. N. Gorb, Composition and substrate-dependent strength of the silken attachment discs in spiders, J. R. Soc., Interface, 2014, 11, 1742-5662, DOI: 10.1098/rsif.2014.0477.

12 C. Apstein, Bau und Funktion der Spinndrüsen der Araneida, Arch. Naturgesch., 1889, 55, 29-74.

13 J. Kovoor and L. Zylberberg, Fine-Structural Aspects of Silk Secretion in a Spider .2. Conduction in the Pyriform Glands, Tissue Cell, 1982, 14, 519-530, DOI: 10.1016/00408166(82)90044-1.

14 W. G. Eberhard, Possible functional significance of spigot placement on the spinnerets of spiders, Journal of Arachnology, 2010, 38, 407-414, DOI: 10.1636/B09-97.1.

15 J. Kovoor and L. Zylberberg, Fine-Structural Aspects of Silk Secretion in a Spider (Araneus-Diadematus) .1. Elaboration in the Pyriform Glands, Tissue Cell, 1980, 12, 547-556, DOI: 10.1016/0040-8166(80)90044-0.

16 J. M. Palmer, F. A. Coyle and F. W. Harrison, Structure and Cyto-Chemistry of the Silk Glands of the Mygalomorph Spider Antrodiaetus-Unicolor (Araneae, Antrodiaetidae), J. Morphol., 1982, 174, 269-274, DOI: 10.1002/jmor.1051740303.

17 F. Vollrath and P. Selden, The role of behavior in the evolution of spiders, silks, and webs, Annu. Rev. Ecol., Evol. Syst., 2007, 38, 819-846, DOI: 10.1146/annurev.ecolsys.37.091305.110221.
$18 \mathrm{H}$. M. Whitney and W. Federle, Biomechanics of plantinsect interactions, Curr. Opin. Plant Biol., 2013, 16, 105111, DOI: 10.1016/j.pbi.2012.11.008.

19 S. D. Eigenbrode, The effects of plant epicuticular waxy blooms on attachment and effectiveness of predatory insects, Arthropod Struct. Dev., 2004, 33, 91-102, DOI: 10.1016/j.asd.2003.11.004.

20 K. Schütt, Wie Spinnen ihre Netze befestigen, Mikrokosmos, 1996, 85, 274-278.

21 D. Jain, V. Sahni and A. Dhinojwala, Synthetic adhesive attachment discs inspired by spider's pyriform silk architecture, J. Polym. Sci., Part B: Polym. Phys., 2014, 52, 553-560, DOI: 10.1002/polb.23453.

22 M. B. Hinman, et al., in Biotechnology of Silk, ed. T. Asakura and T. Miller, Springer, Netherlands, 2014, pp.137-164.

23 P. Geurts, et al. Synthetic Spider Silk Fibers Spun from Pyriform Spidroin 2, A Glue Silk Protein Discovered in OrbWeaving Spider Attachment Discs, Biomacromolecules, 2010, 11, 3495-3503, DOI: 10.1021/bm101002w.

24 P. P. Bhat, et al. Formation of beads-on-a-string structures during break-up of viscoelastic filaments, Nat. Phys., 2010, 6, 625-631, DOI: 10.1038/nphys1682.

25 J. Hu, X. D. Xiao, D. F. Ogletree and M. Salmeron, The structure of molecularly thin films of water on mica in humid environments, Surf. Sci., 1995, 344, 221-236, DOI: 10.1016/0039-6028(95)00858-6.

26 P. B. Miranda, L. Xu, Y. R. Shen and M. Salmeron, Icelike water monolayer adsorbed on mica at room temperature, Phys. Rev. Lett., 1998, 81, 5876-5879, DOI: 10.1103/ PhysRevLett.81.5876.

27 W. H. Voigt, Zur funktionellen Morphologie der Fibroin- und Sericin-sekretion der Seidendrüse von Bombyx mori L., $Z$. Zellforsch. Mikrosk. Anat., 1965, 66, 571-582, DOI: 10.1007/ BF00368247.

28 K. Augsten, K. Weisshart, A. Sponner and E. Unger, Glycoproteins and skin-core structure in Nephila clavipes spider silk observed by light- and electron microscopy, Scanning, 1999, 21, 77.

29 J. O. Wolff, J. M. Schneider and S. N. Gorb, in Biotechnology of silk, ed. T. Asakura and T. Miller, Springer, 2014, ch. 9, pp. 165-177.

30 S. O. Andersen, Amino acid composition of spider silks, Comp. Biochem. Physiol., 1970, 35, 705-711.

31 E. Blasingame, et al. Pyriform Spidroin 1, a Novel Member of the Silk Gene Family That Anchors Dragline Silk Fibers in Attachment Discs of the Black Widow Spider, Latrodectus hesperus, J. Biol. Chem., 2009, 284, 29097-29108, DOI: 10.1074/jbc.M109.021378.

32 A. M. Dranginis, J. M. Rauceo, J. E. Coronado and P. N. Lipke, A biochemical guide to yeast adhesins: Glycoproteins for social and antisocial occasions, Microbiol. Mol. Biol. Rev., 2007, 71, 282-294, DOI: 10.1128/ MMBR.00037-06.

33 E. Hennebert, R. Wattiez and P. Flammang, Characterisation of the Carbohydrate Fraction of the Temporary Adhesive Secreted by the Tube Feet of the Sea Star Asterias rubens, 
Mar. Biotechnol., 2011, 13, 484-495, DOI: 10.1007/s10126010-9319-6.

34 C. P. Brown, et al. Rough Fibrils Provide a Toughening Mechanism in Biological Fibers, ACS Nano, 2012, 6, 19611969, DOI: 10.1021/nn300130q.

35 G. Q. Xu, L. Gong, Z. Yang and X. Y. Liu, What makes spider silk fibers so strong? From molecular-crystallite network to hierarchical network structures, Soft Matter, 2014, 10, 2116-2123, DOI: 10.1039/C3SM52845F.

36 C. P. Brown, et al. The critical role of water in spider silk and its consequence for protein mechanics, Nanoscale, 2011, 3, 3805-3811, DOI: 10.1039/c1nr10502g.
37 V. C. Li, On engineered cementitious composites (ECC): a review of the material and its applications, J. Adv. Concr. Technol., 2003, 1, 215-230.

38 K. L. Johnson, K. Kendall and A. D. Roberts, Surface Energy and Contact of Elastic Solids, Proc. R. Soc. London, Ser. A, 1971, 324, 301-313, DOI: 10.1098/rspa.1971.0141.

39 K. Kendall, Thin-Film Peeling - Elastic Term, J. Phys. D: Appl. Phys., 1975, 8, 1449-1452, DOI: 10.1088/0022-3727/8/13/005.

40 L. Afferrante, G. Carbone, G. Demelio and N. Pugno, Adhesion of Elastic Thin Films: Double Peeling of Tapes Versus Axisymmetric Peeling of Membranes, Tribol. Lett., 2013, 52, 439-447, DOI: 10.1007/s11249-013-0227-6.

41 N. M. Pugno, The theory of multiple peeling, Int. J. Fract., 2011, 171, 185-193, DOI: 10.1007/s10704-011-9638-2. 